\title{
Local structure of copper nitride revealed by EXAFS spectroscopy and reverse Monte Carlo/evolutionary algorithm approach
}

\author{
Janis Timoshenko, Andris Anspoks, Aleksandr Kalinko and \\ Alexei Kuzmin \\ Institute of Solid State Physics, University of Latvia, Kengaraga street 8, LV-1063 \\ Riga, Latvia \\ E-mail: janis.timoshenko@gmail.com
}

\begin{abstract}
Extended x-ray absorption fine structure (EXAFS) spectroscopy combined with reverse Monte Carlo (RMC) and evolutionary algorithm (EA) modelling is used to advance the understanding of the local structure and lattice dynamics of copper nitride $\left(\mathrm{Cu}_{3} \mathrm{~N}\right)$. RMC/EA-EXAFS method provides possibility to probe correlations in the motion of neighboring atoms and allows us to analyze the influence of anisotropic motion of copper atoms in $\mathrm{Cu}_{3} \mathrm{~N}$.
\end{abstract}

\section{Introduction}

Copper nitride $\left(\mathrm{Cu}_{3} \mathrm{~N}\right)$ is a narrow band gap $(0.25-1.90 \mathrm{eV}[1])$ semiconducting material with a cubic anti-ReO $\mathrm{R}_{3}$-type structure composed of corner-shared $\mathrm{NCu}_{6}$ octahedra [2]. It is considered as a metastable material, which loses nitrogen and turns into metallic copper upon temperature increase, and thus is a cheap and promising candidate for write-once memory devices $[3,4,5,6,7]$ : bulk $\mathrm{Cu}_{3} \mathrm{~N}$ is stable at room temperature and decomposes at $300-450{ }^{\circ} \mathrm{C}$, while for thin films the transition to metallic state may occur at significantly lower temperatures (down to $100{ }^{\circ} \mathrm{C}$ ). Besides, copper nitride can be used in a variety of spintronics devices [8, 9], as an active layer in resistive random access memories [10, 11, 12], as a promising cathodic electrocatalyst in alkaline fuel cells [13], in optical lithography for fabrication of microscopic metal links [14, 15] and as a solar energy absorber for photovoltaic and photoelectrochemical solar cells $[1,16]$. The structure of $\mathrm{Cu}_{3} \mathrm{~N}$ is compatible with the structure of some organic molecules, including porphyrins that are considered for applications in molecular-based electronics, thus this material is also interesting due its potential applications in inorganic-organic electronic devices [17]. Deep understanding of the local structure and dynamics of $\mathrm{Cu}_{3} \mathrm{~N}$ is important to fully explain and exploit its unique properties.

A peculiarity of $\mathrm{Cu}_{3} \mathrm{~N}$ lattice dynamics is significant anisotropy in the motion of copper atoms, observed by neutron diffraction [2]. One can also expect that the presence of linear $-\mathrm{N}-\mathrm{Cu}-\mathrm{N}$ - atomic chains in the lattice of $\mathrm{Cu}_{3} \mathrm{~N}$ will result in significant 
correlations in atomic motion, which are crucial for the description of atom dynamics in materials like $\mathrm{ScF}_{3}$ and $\mathrm{ReO}_{3}[18,19]$. Thus, despite the apparent simplicity of $\mathrm{Cu}_{3} \mathrm{~N}$ equilibrium structure, the local structure and local dynamics in this material may be far from trivial. One of a very few approaches that, nevertheless, allow one to study these issues is extended x-ray absorption fine structure (EXAFS) spectroscopy, coupled with advanced simulation-based data analysis methods. To our knowledge, in this study we apply EXAFS spectroscopy at the $\mathrm{Cu}$ K-edge for the investigations of $\mathrm{Cu}_{3} \mathrm{~N}$ for the first time.

EXAFS technique is a modern element-specific method to study the local atomic structure of materials [20]. The analysis of EXAFS data from the first coordination shell around the absorbing atom is based on a well-established procedure [21] and allows one to obtain distributions of distances to the nearest neighbours. At the same time, the experimental EXAFS data, acquired at modern synchrotron radiation sources, contain much more information, especially for crystalline materials. In this case, the total EXAFS consists of contributions from significantly more distant coordination shells (up to $10 \AA$ and further). Besides, the influence of so-called multiple-scattering (MS) effects, which encode the information on many-atom distribution functions and correlations of atomic motion, is often very pronounced in cubic materials such as $\mathrm{Cu}_{3} \mathrm{~N}$. The precise analysis of EXAFS spectra beyond the first coordination shell using conventional methods is, however, often impossible since the total number of parameters, required to completely describe the local structure, is exponentially increasing with the increase of the number of coordination shells, included in the analysis $[22,21]$.

To treat this issue, different simulation-based approaches can be applied: classical and ab initio molecular dynamics (MD) [23, 24, 25, 26], Monte Carlo [27] and reverse Monte Carlo (RMC) methods [28, 29]. All these approaches allow one to obtain a 3D model of material atomic structure, given by a representative set of atomic coordinates in a sufficiently large piece of investigated material. For such model the corresponding theoretical EXAFS spectrum can be calculated using existing ab initio codes [30, 31] and then compared with the experimental data. If the constructed model gives a good agreement between theoretically calculated and experimental EXAFS spectra, then one may expect that the coordinates of atoms in the model represent the atomic configuration in the real material. On the other hand, the agreement between calculated and experimental EXAFS may be used in the case of MD methods for the validation of theoretical simulations [32].

The use of ab initio MD is limited today by high computational costs of the method. Therefore, classical MD simulations are much more popular. They are based on interatomic forces that are set by empirical potentials (so-called force field models [33]). As a result, the accuracy of selected force field model limits the accuracy of the structural model. Moreover, the use of MD methods in the low temperature region, where quantum effects (zero point vibrations) give significant contribution into atomic motion, requires special treatment based on, for example, path-integral method [34]. Note that classical MD method will also fail when the character of chemical bonding in 
a material changes significantly, as, for example, in semiconductor-metal transition. All these issues restrict the use of MD-EXAFS approach.

RMC simulations, in turn, do not require any additional input and do not rely, in principle, on any physical model. In this approach a 3D atomic structure of the material is reconstructed by minimizing the difference between theoretically calculated and experimental structure-related data [28]. Hence this method is quite general and can be applied to the analysis of EXAFS data for a broad range of materials and conditions. The problem of this approach, however, is that it is very time-consuming. Many thousands of iterations are required for the random process that lies at the heart of RMC method to find a structure model that is consistent with experimental data. Moreover, due to significant computational costs of ab initio MS EXAFS simulations for large atomic clusters, the required computational time grows exponentially with the number of coordination shells, included in the analysis. Therefore, in this study we complement the conventional RMC scheme with a powerful evolutionary algorithm (EA) for a very efficient structure model optimization. We have demonstrated recently the use of our approach on the example of perovskite-type $\mathrm{ReO}_{3}$ [19], where the method allowed us to obtain unique information on the local structure, atomic dynamics and correlations in atomic motion in pure and hydrogen intercalated oxide.

In this study we apply RMC/EA-EXAFS approach to the investigations of polycrystalline $\mathrm{Cu}_{3} \mathrm{~N}$ with anti- $\mathrm{ReO}_{3}$ structure. The accuracy of the results, obtained by $\mathrm{RMC} / \mathrm{EA}$ approach, is validated using MD simulations.

\section{Experimental details}

Polycrystalline $\mathrm{Cu}_{3} \mathrm{~N}$ (99.5\% purity) powder was purchased from AlfaAesar and characterized by x-ray diffraction to confirm its single phase composition. The $\mathrm{Cu}_{3} \mathrm{~N}$ powder was deposited on Millipore filter and fixed by Scotch tape for x-ray absorption measurements. The sample thickness $x$ was optimized to obtain the $\mathrm{Cu}$ K-edge jump equal to $\Delta \mu x \approx 1$.

X-ray absorption measurements were performed at the $\mathrm{Cu}$ K-edge $(8979 \mathrm{eV})$ in transmission mode at the HASYLAB/DESY C bending-magnet beamline [35] at room temperature. The storage ring DORIS III operated at $E=4.44 \mathrm{GeV}$ and $I_{\max }=140 \mathrm{~mA}$. The higher order harmonics were reduced by detuning of the doublemonochromator $\mathrm{Si}(111)$ crystals to $60 \%$ of the rocking curve maximum, using the beamstabilization feedback control. The x-ray beam intensity was measured by ionization chambers filled with argon and krypton gases.

The experimental $\mathrm{Cu}$ K-edge EXAFS spectrum $\chi(k) k^{2}$ was extracted using conventional procedure $[36,37]$ and is shown together with its Fourier transform (FT) in Figure 1. Note that contributions from atoms located up to $9 \AA$ from absorbing copper are visible in the FT spectrum. The first two large FT peaks at $\sim 1.5 \AA$ and $\sim 2.5 \AA$ are mostly due to the single-scattering (SS) contributions from the first $(\mathrm{Cu}-\mathrm{N})$ and second $(\mathrm{Cu}-\mathrm{Cu})$ coordination shells of copper, respectively. In principle, they can be 

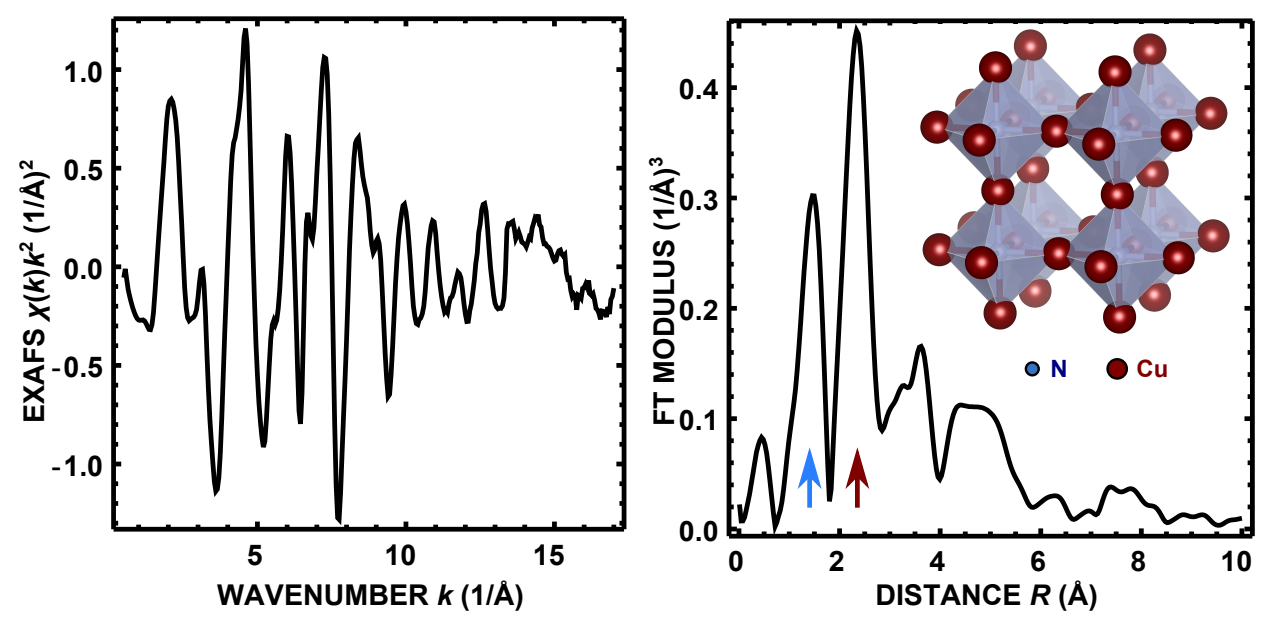

Figure 1. (Color online) Experimental $\mathrm{Cu}$ K-edge EXAFS spectrum and its Fourier transform (FT) at room temperature. A fragment of crystal structure of cubic $\mathrm{Cu}_{3} \mathrm{~N}$ is shown in the inset [2].

analysed using conventional EXAFS fitting approaches [21, 36]. Further FT peaks are due to a complex superposition of SS contributions from outer coordination shells and all MS contributions, which are expected to be important in perovskite-type structure due to the presence of linear atomic chains [38]. In fact, peaks at about $3.5 \AA$ and $5 \AA$ are mostly due to the multiple-scattering contributions within $\mathrm{NCu}_{6}$ octahedra and within $-\mathrm{N}-\mathrm{Cu}-\mathrm{N}-$ linear chains. The analysis of these contributions using conventional approaches would be a challenging problem.

\section{Reverse Monte Carlo/evolutionary algorithm simulations}

To treat experimental $\mathrm{Cu}$ K-edge EXAFS data we employ an enhanced RMC-type approach, based on the use of evolutionary algorithm [19]. The combined RMC/EA scheme allows us to perform accurate analysis of EXAFS data from distant coordination shells of crystalline materials, taking into account static and thermal disorder as well as MS effects. As in the conventional RMC method, the 3D structure model of a material in our approach is optimized via the consequent proposal of random changes of atomic coordinates, and the agreement between the experimental and configuration-averaged EXAFS spectra, calculated for the proposed structure model, is used as the only criterion for the acceptance or rejection of proposed move. This configuration-averaged EXAFS spectrum is obtained from ab initio MS calculations, performed at each iteration by the FEFF8 code [30].

Implementation of the evolutionary algorithm significantly improves the convergence of the RMC optimization scheme and reduces the number of required iterations. In this case we use not just one, but several (32 in this study) structure models of the material simultaneously. The information exchange between the simulated structure models allows us to explore the configurational space more efficiently and thus the opti- 
mal solution - the average atomic configuration that gives the best possible description of experimental data - is found much faster [19].

For RMC/EA calculations an initial structure model should be provided. We started with the equilibrium structure, known from diffraction experiment [2], with some small random shift for all atoms applied, and used $4 \times 4 \times 4$ supercell, containing 256 atoms, with periodic boundary conditions. The value of lattice constant $a_{0}$ was taken from diffraction studies, and was not refined during the simulations.

At each RMC/EA iteration the theoretical EXAFS spectra are calculated for each absorbing $\mathrm{Cu}$ atom in the supercell using ab initio self-consistent real-space MS FEFF8 code [30], taking into account MS contributions with up to eight backscatters involved. Then the configuration-averaged EXAFS spectrum is obtained. The calculation of the cluster potential is performed only once (at the beginning of simulations) for the mean crystallographic $\mathrm{Cu}_{3} \mathrm{~N}$ structure [2], thus neglecting its small variation due to thermal disorder. The complex exchange-correlation Hedin-Lundqvist potential and default values of muffin-tin radii as provided within the FEFF8 code [30] are employed.

The obtained configuration-averaged EXAFS spectrum is compared with the experimental one. Instead of comparing them directly in $k$-space or in Fourier transform $R$-space, we apply the Morlet wavelet transform $[39,40]$ for both spectra

$$
w(k, R)=\sqrt{R / R_{0}} \int_{-\infty}^{+\infty} \chi\left(k^{\prime}\right) \varphi\left(\left(R / R_{0}\right)\left(k^{\prime}-k\right)\right) d k^{\prime},
$$

where $\chi(k)$ is the corresponding EXAFS spectrum, $\varphi(k)=\exp \left(-2 i R_{0} k\right) \exp \left(-\sigma_{0}^{2} k^{2}\right)$ is so-called mother wavelet function with the parameters $R_{0}$ and $\sigma_{0}$ that can improve the resolution of the transform in $k$-space by reducing the resolution in $R$-space, and vice versa. A significant advantage of using wavelet transform for EXAFS analysis is that it accounts for and shows the behaviour of EXAFS signal simultaneously in $k$ and $R$-space, thus stabilizing the solution obtained during the simulation/fit. Besides, wavelet transform allows one to discriminate between contributions of different atoms, since it is known, for instance, that light elements contribute more strongly at lower $k$-values than heavier elements. Indeed, contributions of $\mathrm{Cu}-\mathrm{N}$ and $\mathrm{Cu}-\mathrm{Cu}$ pairs are clearly separated in wavelet transform image in Figure 2.

A difference between the model and RMC EXAFS spectra can be calculated as

$$
\xi_{k, R}=\frac{\left\|w_{\text {tot }}(k, R)-w_{\exp }(k, R)\right\|_{2}}{\left\|w_{\exp }(k, R)\right\|_{2}},
$$

where $w_{\text {tot }}$ and $w_{\text {exp }}$ are the wavelet images of the calculated and experimental EXAFS spectra, respectively, and $\|\ldots\|_{2}$ denotes the Euclidean norm [19]. Here wavelet transforms and norm of their difference were calculated in $k$-space range from $3.0 \AA^{-1}$ to $17 \AA^{-1}$ and in $R$-space range from $R_{\min }=1.0 \AA$ to $R_{\max }=6.0 \AA$ (see Figure 2 ). Mother wavelet functions parameters were $\sigma_{0}=1.0 \AA$ and $R_{0}=\left(R_{\min }+R_{\max }\right) / 2$.

$\xi_{k, R}$ thus allows us to quantify the fitness of each of 32 simulated atomic configurations. By applying evolutionary operators and random atomic displacements to all atoms in all supercells, as described in [19], at each iteration from the previous 

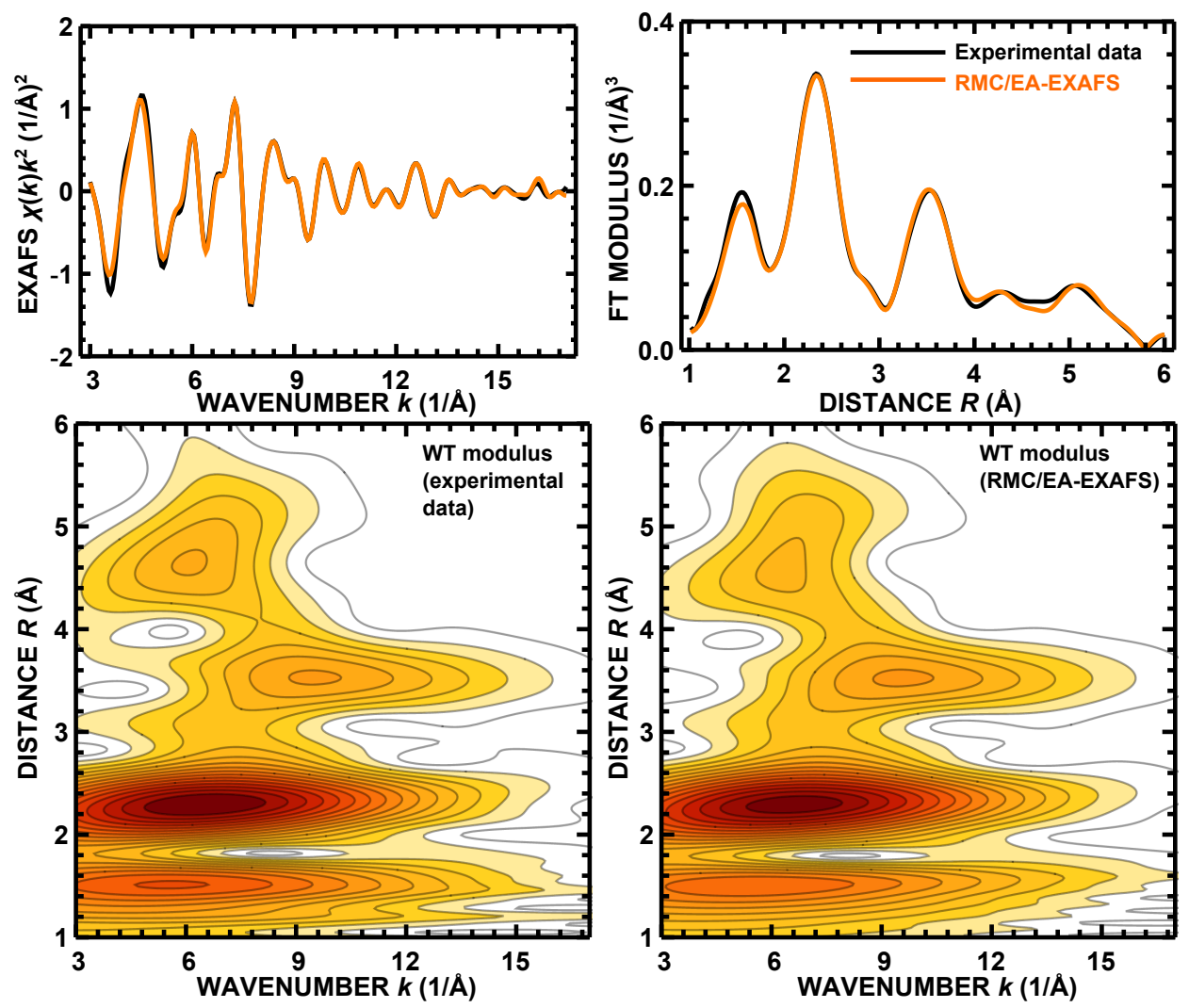

Figure 2. (Color online) Comparison of experimental $\mathrm{Cu}$ K-edge EXAFS and the theoretical EXAFS, obtained in RMC/EA simulations: their Fourier filtered EXAFS and Fourier transforms (upper panels) and Morlet wavelet transforms (bottom panels).

generation of atomic configurations one obtains a new set of 32 configurations, for which the difference between theoretical and experimental EXAFS spectra will, on average, be lower than for the former generation. By repeating this process a good agreement between both spectra can be obtained after several hundreds of iterations. At this moment the simulated atomic configurations will resemble the positions of atoms in the real sample. In more details the used RMC/EA scheme is presented in our recent paper [19].

The results of such simulations for room temperature $\mathrm{Cu}$ K-edge EXAFS in $\mathrm{Cu}_{3} \mathrm{~N}$ are shown in Figures 2 and 3. In Figure 2 the experimental EXAFS is compared with the calculated EXAFS for the final atomic configuration, obtained in RMC/EA simulation and shown in Figure 3 (left panel). One can ascertain that the structure model, obtained using RMC/EA approach, is in a good agreement with the experimental data. Also our model - stoichiometric, defect-free crystal - does not contradict to the available experimental EXAFS data, i.e., there is no evidence of $\mathrm{Cu}_{3} \mathrm{~N}$ decomposition or metallization.

From a set of atomic coordinates, obtained in RMC/EA simulation, one can calculate partial radial distribution functions (RDFs) $g(R)$ for the $\mathrm{Cu}-\mathrm{N}$ and $\mathrm{Cu}-\mathrm{Cu}$ atom pairs, as shown in Figure 3 (right panel) and, in principle, estimate structural 

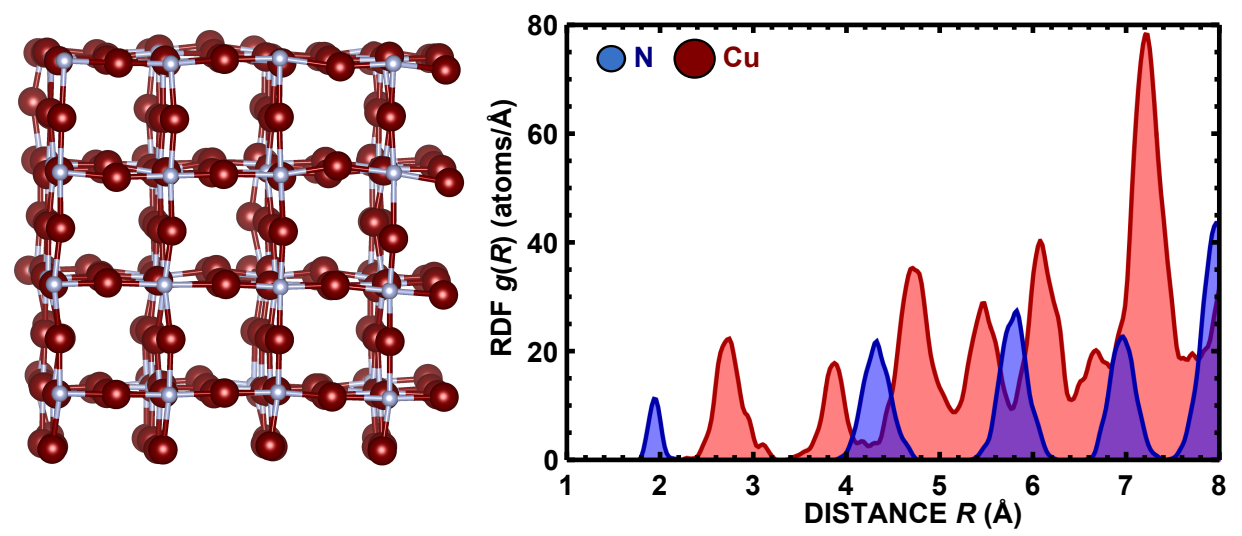

Figure 3. (Color online) Final $\mathrm{Cu}_{3} \mathrm{~N}$ structure model, obtained in RMC/EA simulations for experimental $\mathrm{Cu}$ K-edge EXAFS data, and corresponding partial radial distribution functions (RDFs) around copper atoms.

parameters such as average interatomic distances (RDF peak positions), distance variations (so-called mean-square radial displacement factors (MSRDs) characterizing the widths of RDF peaks), bonding angles distributions, etc. for any coordination shell with the radius smaller than the half-length of the used supercell. It is obvious that the accuracy of structural parameter values for the nearest coordination shells will be higher than for distant coordination shells. The contribution of the nearest shells into the total EXAFS spectrum is much larger, and imprecision in the average interatomic distances or relative displacements for the nearest shells would result in a significant discrepancy between experimental and calculated EXAFS. The set of structural parameters that can be reliably estimated from the results of RMC/EA-EXAFS simulations and their accuracy will be different for different materials and different absorption edges. For instance, the presence of strong MS contribution improves sensitivity of our approach to bonding angles, while the presence of heavy backscatter in the system allows one to probe more distant coordination shells. Therefore, before using the result of RMC/EA simulations for the analysis of distant coordination shells, it is important to validate that RMC/EA approach provides sufficient sensitivity for such analysis. For this purpose the RMC/EA simulations for model EXAFS data, i.e., for EXAFS spectrum, for which the corresponding structure is known in advance, can be helpful. To generate such model data, we employ here classical molecular dynamics simulations.

\section{Molecular dynamics simulations}

To our knowledge, no force-field models exist for $\mathrm{Cu}_{3} \mathrm{~N}$ in the literature. The development of a force field model, which would give an accurate description of local structure of the material, is a complex task and deserves a separate study. In our case, however, our intent is to use MD simulations just to validate RMC/EA procedure. Therefore we do not strive here for a precise result, and to set interatomic forces in $\mathrm{Cu}_{3} \mathrm{~N}$ we use a simple potential model close to that employed by us earlier in MD- 
Table 1. Buckingham potential parameters for the $\mathrm{Cu}-\mathrm{N}$ and $\mathrm{Cu}-\mathrm{Cu}$ atom pairs used in molecular dynamics simulations.

\begin{tabular}{lccc}
\hline Pair of atoms & $A(\mathrm{eV})$ & $\rho(\AA)$ & $C\left(\mathrm{eV} \AA^{6}\right)$ \\
\hline $\mathrm{Cu}-\mathrm{N}$ & 78.53 & 0.6241 & 0.0 \\
$\mathrm{Cu}-\mathrm{Cu}$ & 6262 & 0.2525 & 0.0 \\
\hline
\end{tabular}

Table 2. Covalent-exponential potential parameters for the $\mathrm{Cu}-\mathrm{N}$ and $\mathrm{Cu}-\mathrm{Cu}$ atom pairs used in molecular dynamics simulations.

\begin{tabular}{lccc}
\hline Pair of atoms & $D(\mathrm{eV})$ & $a\left(\AA^{-1}\right)$ & $r_{0}(\AA)$ \\
\hline $\mathrm{Cu}-\mathrm{N}$ & 4.378 & 5.231 & 1.910 \\
$\mathrm{Cu}-\mathrm{Cu}$ & 2.375 & 3.473 & 2.700 \\
\hline
\end{tabular}

EXAFS study of $\mathrm{ReO}_{3}$ [41]. Our interaction potential consists of the Buckingham and covalent-exponential potentials [42]. Additionally, the Coulomb interactions were accounted using the effective ion charges $+0.5 e$ for copper and $-1.5 e$ for nitrogen atoms in agreement with ab initio calculations of electronic structure of $\mathrm{Cu}_{3} \mathrm{~N}$ [43]. The parameters of the Buckingham potential and covalent-exponential potential were optimized to reproduce as close as possible the lattice constant, bulk modulus and phonon frequencies of $\mathrm{Cu}_{3} \mathrm{~N}$. Optimized values of the Buckingham potential are given in Table 1, while the values of the covalent exponential potential are given in Table 2. Note that parameter $C$ for Buckingham potential was found to be 0 in our force field model, hence it also may be called Born-Mayer potential [44].

Classical molecular dynamics (MD) simulations were performed using the GULP code $[33,42]$ in the canonical (NVT) ensemble with periodic boundary conditions. The simulation box had a size of the $5 a_{0} \times 5 a_{0} \times 5 a_{0}$ supercell including 500 atoms. NoséHoover thermostat [45] was used to keep the required average temperature during each simulation. The Newton's equations of motion were integrated using the Verlet leapfrog algorithm [46] with a time step of $0.5 \mathrm{fs}$. The equilibration and production times were 20 ps each. 4000 atomic configurations, accumulated during each production run, were then used to calculate configuration-averaged EXAFS spectrum, using the same approach as used for EXAFS calculations during RMC/EA simulations. Thus obtained model EXAFS spectrum (MD-EXAFS) is shown in Figure 4 together with its Fourier and wavelet transforms.

Next, we used the MD-EXAFS spectrum as an input for RMC/EA simulations with the same parameters and fitting ranges as used previously for the analysis of the experimental EXAFS data. The $\mathrm{Cu} \mathrm{K}$-edge EXAFS spectrum corresponding to final configuration, obtained in RMC/EA simulations for model data, is shown in Figure 4. The agreement between MD-EXAFS and RMC/EA-EXAFS data is good, indicating that RMC/EA method was able to find a structure model, consistent with the model EXAFS data. Note that there is small discrepancy between the model data 

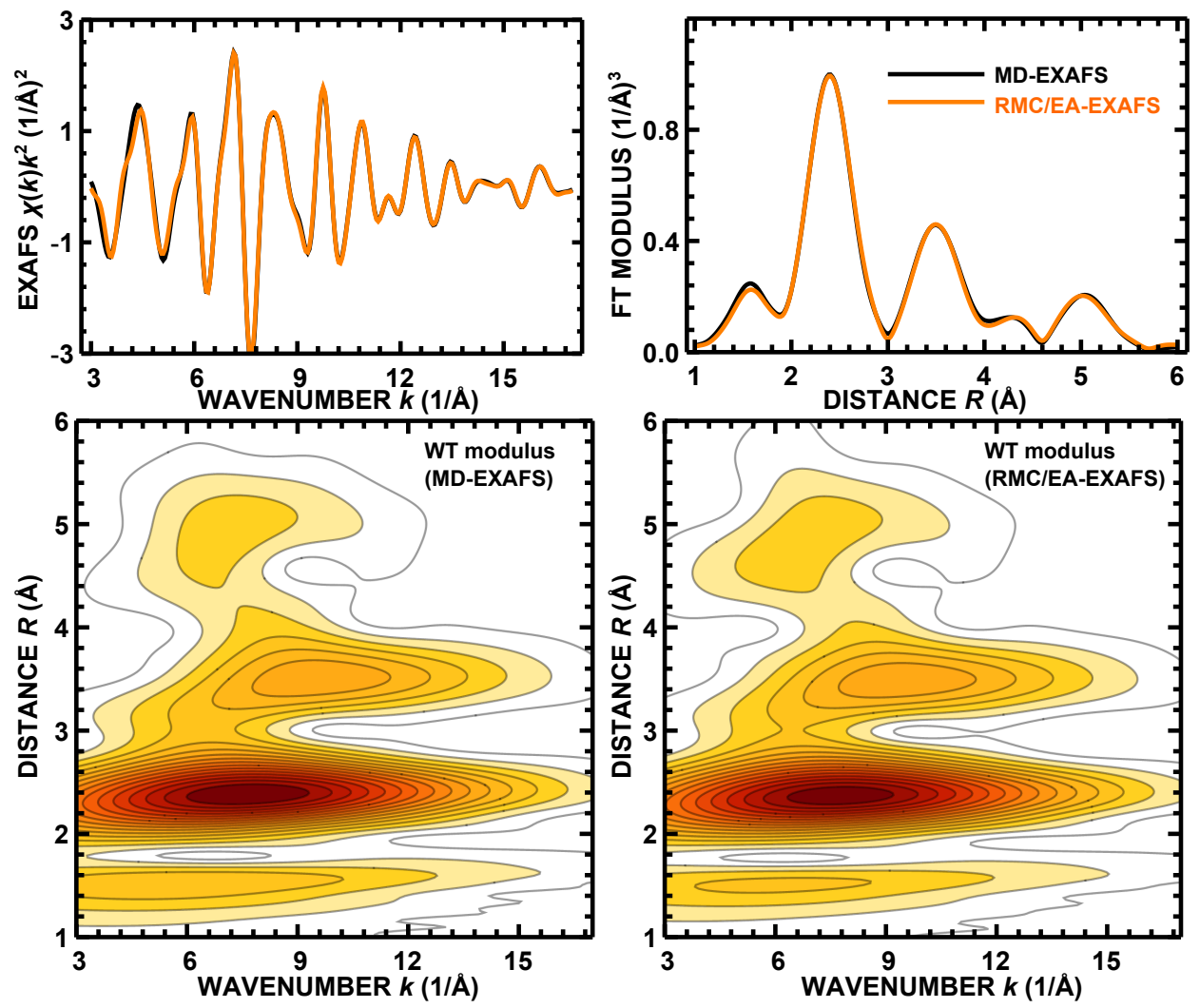

Figure 4. (Color online) Comparison of model $\mathrm{Cu}$ K-edge EXAFS spectrum, obtained in MD simulations, and the result of its RMC/EA reconstruction: Fourier filtered EXAFS spectra and their Fourier transforms (upper panels) and Morlet wavelet transforms (bottom panels).

and RMC/EA-EXAFS spectrum in the first coordination shell: the amplitude of the first peak in FT is lower for RMC/EA result. As will be commented below, it is an evidence that RMC/EA approach overestimates slightly the structural disorder in this case. Note that similar discrepancies between the model EXAFS data and the result of $\mathrm{RMC} / \mathrm{EA}$ simulations were obtained also for RMC/EA fitting of the experimental $\mathrm{Cu}$ K-edge EXAFS spectrum of $\mathrm{Cu}_{3} \mathrm{~N}$ (Figure 3).

Comparison of structural parameters, obtained from RMC/EA modelling for the model EXAFS data, with their known values allows one to estimate the uncertainties of the analysis also for the experimental data.

\section{Results and discussion}

Structural parameters of the MD model are compared with the results of RMC/EA reconstruction for the first 16 coordination shells around copper in Figure 5. Each point in the graphs corresponds to a specific coordination shell, and we show separately structural parameters for pairs of atoms that are located within a single $-\mathrm{N}-\mathrm{Cu}-\mathrm{N}-$ chain and for atom pairs located off this chain. Here we focus on two types of parameters: (i) MSRD factors, which characterize the variation in the interatomic distances due to atom 

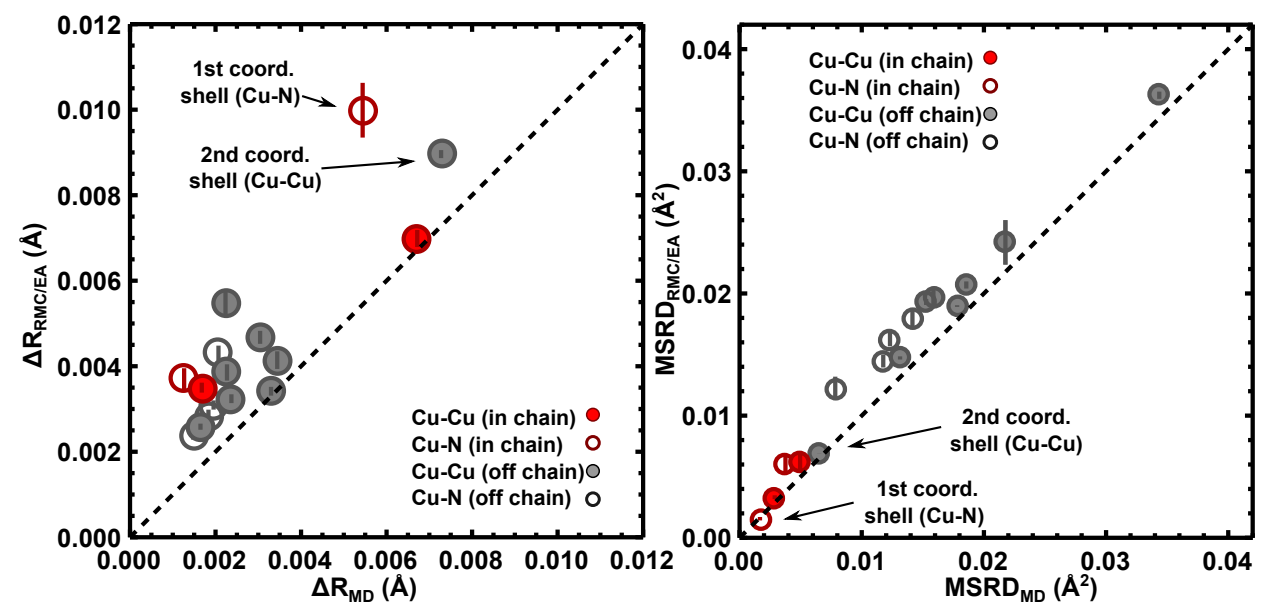

Figure 5. (Color online) Comparison of structural parameters of MD model (..MD) with the results of RMC/EA reconstruction (..RMC/EA ): relative average interatomic distances with respect to crystallographic values $\Delta R=R-R_{\text {eq }}$ (see equation (3)) and MSRD factors for the first 16 coordination shells.

motion in the direction of interatomic bond and (ii) a difference $\Delta R$ between the average interatomic distance $R$ and the distance $R_{\text {eq }}$ between the average positions of atoms. Note that due to the motion of atoms in the direction, perpendicular to interatomic bond, average interatomic distances $R$, measured by EXAFS, are always larger than crystallographic distances $R_{\text {eq }}$ between the average positions of atoms [47]. Hence $\Delta R=R-R_{\mathrm{eq}}$ can be used to characterize the amplitude $\left\langle u_{\perp}^{2}\right\rangle$ of atomic displacement in the direction perpendicular to interatomic bond [47]. It is known, in fact, that average interatomic distance $R$, as probed by EXAFS, and perpendicular MSRD factor $\left\langle u_{\perp}^{2}\right\rangle$ are related by equation $[47]$

$$
R \approx R_{\mathrm{eq}}+\frac{\left\langle u_{\perp}^{2}\right\rangle}{2 R_{\mathrm{eq}}} .
$$

RMC/EA method provides values slightly larger than those for the original MD structure both for MSRD and $\Delta R$ (Figure 5). This finding is consistent with the observation that RMC-type methods tend to converge of all possible structure models that agree with experimental data to the one that have the maximal disorder. For all coordination shells the difference between values of $\Delta R$ parameters, obtained in $\mathrm{RMC} / \mathrm{EA}$ simulations, and true values for MD model is smaller than $0.005 \AA$. It may seem surprising that the largest differences are observed in the values of $\Delta R$ for the first coordination shell, which gives the most significant contribution to the EXAFS spectrum. This finding, however, is in agreement with Equation (3): overestimation in disorder in the direction, orthogonal to interatomic bonds, will affect more the lengths of short bonds. The longer bonds are less affected by the disorder effects, and accuracy of their lengths determination is closely related to the accuracy of lattice constants values, used for RMC/EA simulations.

The differences between the values of MSRD factors, obtained in RMC/EA simulations, and the values for MD model are smaller than $0.001 \AA^{2}$ for two nearest 
Table 3. Structural parameters (MSD factors and $\mathrm{Cu}-\mathrm{N}-\mathrm{Cu}$ angle $\varphi$ ) for MD model and for final configuration, obtained in RMC/EA simulations of model data. See details in text.

\begin{tabular}{lcc}
\hline Parameter & $\mathrm{MD}$ & $\mathrm{RMC} / \mathrm{EA}$ for MD \\
\hline $\mathrm{MSD}^{11}(\mathrm{~N})=\mathrm{MSD}^{22}(\mathrm{~N})=\mathrm{MSD}^{33}(\mathrm{~N})\left(\AA^{2}\right)$ & 0.00458 & $0.0045(1)$ \\
$\mathrm{MSD}^{11}(\mathrm{Cu})\left(\AA^{2}\right)$ & 0.00288 & $0.0034(4)$ \\
$\mathrm{MSD}^{22}(\mathrm{Cu})=\mathrm{MSD}^{33}(\mathrm{Cu})\left(\AA^{2}\right)$ & 0.00106 & $0.010(1)$ \\
$\varphi\left(^{\circ}\right)$ & 173.0 & $170.9(3)$ \\
\hline
\end{tabular}

coordination shells and for $\mathrm{Cu}-\mathrm{Cu}$ pairs, located within the $-\mathrm{N}-\mathrm{Cu}-\mathrm{N}-$ chain: these groups of atoms contribute strongly to the total EXAFS spectrum, hence our analysis is quite sensitive to them. Systematic errors of MSRD factors for other coordination shells may be several times larger, since the values of MSRD factors themselves are large, which results in reduced amplitudes of corresponding contributions to the total EXAFS spectrum. Note here that small values of relative displacements of atoms in the nearest coordination shells are ensured by correlations in atom motion, which, naturally, decrease with the increase of corresponding interatomic distance.

Note that the test results, presented here, allow one to estimate systematic errors of RMC/EA fitting procedure only, and do not account, for instance, for systematic errors due to EXAFS extraction procedure, sample homogeneity issues, monochromator stability, etc. Also imprecisions due to inaccuracies of FEFF calculations were not taken into account here, since the same FEFF theory [30] was used both for generation of MDEXAFS model data and for RMC/EA reconstruction. It is believed that FEFF based analysis of experimental EXAFS data provides interatomic distances with accuracy about $0.01 \AA$, thus systematic errors, introduced by RMC/EA procedure, are still smaller than the ones, introduced by the use of current FEFF theory.

Besides MSRDs and interatomic distances, there are several additional important structural parameters. Mean square displacement (MSD) factors characterize the absolute deviations of atoms from their equilibrium positions. These quantities are usually obtained from diffraction experiments, but simulation-based EXAFS analysis can also be used for this purpose: indirect information about MSD factors is encoded in angular dependencies of MS contributions and in contributions of distant coordination shells [48], where the motion of atoms is almost uncorrelated, hence their MSRD factors can be expressed just as a sum of corresponding MSDs. These quantities are summarized in Table 3. Motion of nitrogen atoms in $\mathrm{Cu}_{3} \mathrm{~N}$ is isotropic, hence their MSD values along $-\mathrm{Cu}-\mathrm{N}-\mathrm{Cu}-$ chain direction and in two orthogonal directions (denoted further as $\mathrm{MSD}^{11}(\mathrm{~N}), \mathrm{MSD}^{22}(\mathrm{~N})$ and $\mathrm{MSD}^{33}(\mathrm{~N})$ ) are equal. This MSD value is estimated reliably in the structure model, obtained in RMC/EA calculations for model MD-EXAFS data: the value of $\mathrm{MSD}^{11}(\mathrm{~N})=\mathrm{MSD}^{22}(\mathrm{~N})=\mathrm{MSD}^{33}(\mathrm{~N})$ agrees with the result of RMC/EA simulation within error bars. The motion of copper atoms is significantly anisotropic, 
Table 4. Structural parameters for $\mathrm{Cu}_{3} \mathrm{~N}$, obtained by RMC/EA analysis of experimental room temperature EXAFS data and in diffraction experiments.

\begin{tabular}{lccc}
\hline Parameter & RMC/EA & Diffraction [2] & Diffraction [49] \\
\hline $\operatorname{MSD}^{11}(\mathrm{~N})=\mathrm{MSD}^{22}(\mathrm{~N})=\mathrm{MSD}^{33}(\mathrm{~N})\left(\AA^{2}\right)$ & $0.006(1)$ & $0.0058(1)$ & $0.0060(8)$ \\
$\mathrm{MSD}^{11}(\mathrm{Cu})\left(\AA^{2}\right)$ & $0.008(1)$ & $0.00246(6)$ & $0.0048(6)$ \\
$\mathrm{MSD}^{22}(\mathrm{Cu})=\mathrm{MSD}^{33}(\mathrm{Cu})\left(\AA^{2}\right)$ & $0.017(2)$ & $0.0124(4)$ & $0.012(1)$ \\
$\varphi\left({ }^{\circ}\right)$ & $169.9(3)$ & - & - \\
\hline
\end{tabular}

and $\mathrm{MSD}^{11}(\mathrm{Cu})$ factor, corresponding to their motion along $-\mathrm{Cu}-\mathrm{N}-\mathrm{Cu}-$ chain direction, is significantly smaller than $\operatorname{MSD}^{22}(\mathrm{Cu})$ and $\operatorname{MSD}^{33}(\mathrm{Cu})$ factors. This anisotropy is well reproduced by our $\mathrm{RMC} / \mathrm{EA}$ method. Note, however, that $\mathrm{MSD}^{11}(\mathrm{Cu})$ value is slightly overestimated.

Finally, another important parameter is the angle $\varphi$ between neighboring $\mathrm{N}-\mathrm{Cu}$ and $\mathrm{Cu}-\mathrm{N}$ bonds in $-\mathrm{N}-\mathrm{Cu}-\mathrm{N}$ - chain (i.e., the tilting angle of $\mathrm{NCu}_{6}$ octahedra). For equilibrium structure of $\mathrm{Cu}_{3} \mathrm{~N}$ this angle is equal to $180^{\circ}$. Due to thermal motion of atoms, however, locally the average value of this angle is always smaller, and will be the smaller, the larger are displacements of $\mathrm{Cu}$ atoms in the direction orthogonal to the $-\mathrm{N}-\mathrm{Cu}-\mathrm{N}-$ chain. The value of $\varphi$, obtained in $\mathrm{RMC} / \mathrm{EA}$ simulations, is about $2^{\circ}$ smaller than the one for the MD model, indicating once again that the disorder of $\mathrm{Cu}_{3} \mathrm{~N}$ is slightly overestimated in RMC/EA simulations.

Further we will discuss the results, obtained by RMC/EA method for the experimental $\mathrm{Cu}$ K-edge EXAFS data for $\mathrm{Cu}_{3} \mathrm{~N}$, measured at room temperature.

Variations of $\Delta R$ and MSRD factors for different $\mathrm{Cu}-\mathrm{Cu}$ and $\mathrm{Cu}-\mathrm{N}$ pairs as a function of corresponding crystallographic distance $R_{\text {eq }}$ are shown in Figure 6, while the obtained values of MSD factors and average $\varphi$ angle are given in Table 4. MSD factors, obtained by our RMC/EA-EXAFS analysis, are compared here with the results of neutron diffraction studies [2]. MSD values for nitrogen, obtained by two methods, agree within error bars, and also significant anisotropy of copper atom vibrations is clearly evidenced by both methods. Our analysis, however, suggests significantly larger values of MSD both in the direction of $-\mathrm{N}-\mathrm{Cu}-\mathrm{N}-$ chain and in the orthogonal directions. Note that the difference between copper MSD values, obtained by our EXAFS analysis and by neutron diffraction, exceeds significantly the uncertainty of our method, estimated in our study of model EXAFS data, and would require a separate investigation in the future.

In Figure 6 (right panel) we compare MSRD factors for the $\mathrm{Cu}-\mathrm{N}$ bond with the sum of corresponding MSD factors for copper and nitrogen atoms. Note that the knowledge of MSRD and MSD factors for the two atoms $\mathrm{A}$ and $\mathrm{B}$ allows one to estimate correlation effects: $\operatorname{MSRD}(\mathrm{A}, \mathrm{B})=\operatorname{MSD}(\mathrm{A})+\operatorname{MSD}(\mathrm{B})-2 \operatorname{cov}(\mathrm{A}, \mathrm{B})$, where $\operatorname{cov}(\mathrm{A}, \mathrm{B})$ is the covariance of displacements of atoms $\mathrm{A}$ and $\mathrm{B}$ and accounts for correlation in atom motion [20]. 

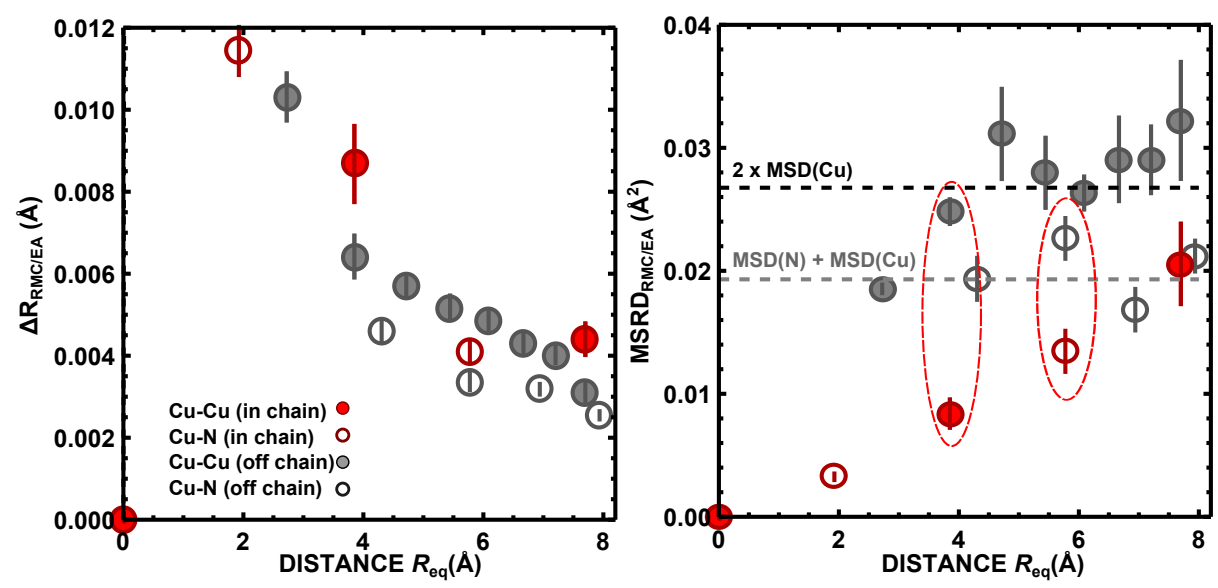

Figure 6. (Color online) Results of the analysis of the experimental $\mathrm{Cu}$ K-edge EXAFS data: relative interatomic distances with respect to crystallographic values $\Delta R=R-R_{\text {eq }}$ (see equation (3)) and MSRD factors for different $\mathrm{Cu}-\mathrm{Cu}$ and $\mathrm{Cu}-\mathrm{N}$ pairs as functions of corresponding crystallographic distance $R_{\text {eq }}$. Dashed lines in the right panel - sum of corresponding copper and nitrogen MSD factors (as copper MSD the averaged value of $\operatorname{MSD}^{11}(\mathrm{Cu}), \mathrm{MSD}^{22}(\mathrm{Cu})$ and $\operatorname{MSD}^{33}(\mathrm{Cu})$ is used).

It is instructive to examine MSRD values for atom pairs located within single linear $-\mathrm{N}-\mathrm{Cu}-\mathrm{N}-$ chain and to compare them with MSRDs for atoms in the off-chain positions. Note that the MSRD factors for atom pairs located along a single chain are relatively small. Striking examples of the difference between off-chain and in-chain MSRDs can be seen in the 3rd and 7th coordination shells of copper atoms $\left(R_{\text {eq }}=3.850 \AA\right.$ and $R_{\mathrm{eq}}=5.775 \AA$, correspondingly, as highlighted in Figure 6 (right panel)). Note that there are six copper atoms in the 3rd coordination shell of each copper. While all these six copper atoms have the same equilibrium distance to central copper, two of them are located within a $-\mathrm{N}-\mathrm{Cu}-\mathrm{N}-$ chain with the absorbing copper atom, while the remaining four are located in the off-chain positions. Despite the fact that $R_{\text {eq }}$ value is the same for all six copper atoms in the 3rd coordination shell, the MSRD factors for these two groups of atoms are significantly different. Similar findings can be done for the 7th coordination shell, consisting of two nitrogen atoms in the in-chain positions and eight nitrogen atoms in the off-chain positions.

Differences of MSRDs for in-chain and off-chain atoms can be a result of smaller amplitudes of copper oscillations in the direction along the chain, as well as due to differences in the correlations of atom motion for in-chain and off-chain pairs. Taking into account that the MSRDs for off-chain pairs are close to the sum of corresponding MSDs for copper and nitrogen atoms and show weak dependence on interatomic distance, while the MSRDs for in-chain pairs grow with the increase of interatomic distance, one can conclude that the correlation of atom motion in the direction of $-\mathrm{N}-$ $\mathrm{Cu}-\mathrm{N}$ - chain plays an important role for dynamics of in-chain atoms. Such directional dependence of atomic interactions in $\mathrm{Cu}_{3} \mathrm{~N}$ suggests the presence of pronounced covalent bonding in this material, as predicted by ab initio studies of its electronic structure [43]. 

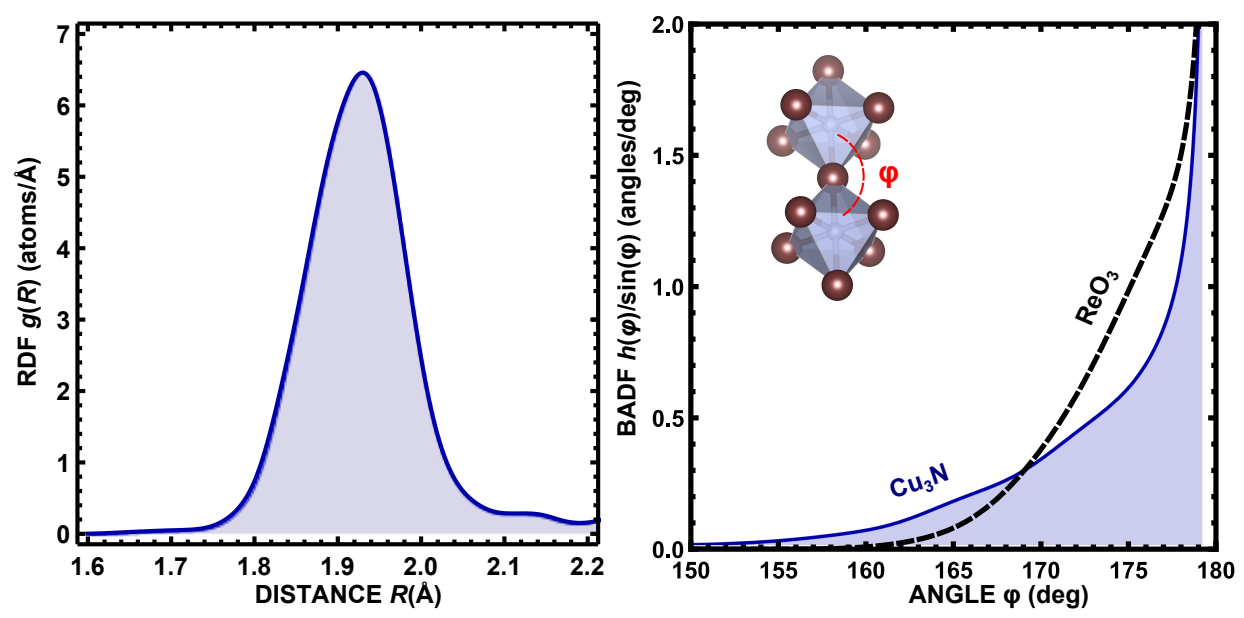

Figure 7. (Color online) Results of the analysis of the experimental $\mathrm{Cu}$ K-edge EXAFS data: distribution of $\mathrm{Cu}-\mathrm{N}$ interatomic distances in the first coordination shell around $\mathrm{Cu}$ atoms and bonding angle distribution function (BADF) of $\mathrm{N}-\mathrm{Cu}-\mathrm{N}$ angles $\varphi$. The latter is defined as probability density $h(\varphi)$ to encounter angle with value $\varphi$, divided by $\sin (\varphi)$. For comparison, the results for $\mathrm{ReO}_{3}[19]$ are also shown.

Note also that such behaviour of the MSRD factors is similar to the one, observed by us for crystalline $\mathrm{ReO}_{3}$ [50]. For more detailed analysis of interatomic interactions in $\mathrm{Cu}_{3} \mathrm{~N}$ temperature-dependent EXAFS studies of $\mathrm{Cu}_{3} \mathrm{~N}$ should be carried out.

Note that we do not observe any significant differences in the behavior of $\Delta R$ parameters within the precision of our analysis for in-chain and off-chain atom pairs (Figure 6 (left panel)). In both cases the dependencies of $\Delta R$ parameter are in a qualitative agreement with Equation (3). This result suggests that there are no significant correlations in the atom motion in the direction orthogonal to $-\mathrm{N}-\mathrm{Cu}-\mathrm{N}-$ chain direction.

Finally, let us discuss our results regarding the angle between neighbouring $\mathrm{NCu}_{6}$ octahedra. Its value has been a subject of controversy in the literature [2, 49]. Our results indicate that the cubic structure of $\mathrm{Cu}_{3} \mathrm{~N}$ (where the angle between octahedra, formed by equilibrium positions of $\mathrm{Cu}$ and $\mathrm{N}$ atoms, as seen by diffraction studies [2], is $180^{\circ}$ ) is fully consistent with the experimental EXAFS data. At the same time, the average $\mathrm{N}-\mathrm{Cu}-\mathrm{N}$ angle $\varphi$ is smaller than $180^{\circ}$ due to oscillations of copper atoms in the directions, orthogonal to $-\mathrm{N}-\mathrm{Cu}-\mathrm{N}-$ chains. In fact, our results suggest that this effect is very pronounced in $\mathrm{Cu}_{3} \mathrm{~N}$. The obtained distribution of $\mathrm{N}-\mathrm{Cu}-\mathrm{N}$ bonding angles is shown in Figure 7, and, when compared with the corresponding distribution for $\mathrm{Re}-\mathrm{O}-$ Re angles in $\mathrm{ReO}_{3}$ [19], one can conclude that it is much broader in $\mathrm{Cu}_{3} \mathrm{~N}$. Hence the average $\mathrm{N}-\mathrm{Cu}-\mathrm{N}$ angle value $\left(\sim 170^{\circ}\right)$ is significantly smaller than that in $\mathrm{ReO}_{3}\left(\sim 174^{\circ}\right)$, indicating the presence of large anharmonic vibrations of copper in $\mathrm{Cu}_{3} \mathrm{~N}$. Tentatively they can be attributed to the tilting motion of $\mathrm{NCu}_{6}$ octahedra, since the relatively small value of MSRD factor for the shortest $\mathrm{Cu}-\mathrm{N}$ bond implies rigidity of $\mathrm{NCu}_{6}$ unit. Note that it is pointed out in [51], from electronic structure point of view it should 
be easier to tilt the $\mathrm{NCu}_{6}$ octahedra in $\mathrm{Cu}_{3} \mathrm{~N}$ than $\mathrm{ReO}_{6}$ octahedra in $\mathrm{ReO}_{3}$, and our results support this finding. Such strong anharmonic motion of copper atoms should result in non-Gaussian shape of the RDF for the first coordination shell $(\mathrm{Cu}-\mathrm{N}$ bond), and, indeed, it is clearly seen in our results, obtained by RMC/EA method (Figure 7 (left panel)).

\section{Conclusions}

In this paper we report on the first EXAFS study of the local structure in polycrystalline $\mathrm{Cu}_{3} \mathrm{~N}$ at room temperature. The experimental $\mathrm{Cu}$ K-edge EXAFS data were analysed by advanced simulation-based method, involving the use of reverse Monte Carlo, evolutionary algorithm and wavelet transform approaches. We have demonstrated that the RMC/EA-EXAFS method is sensitive enough to analyze the local structure of $\mathrm{Cu}_{3} \mathrm{~N}$ within up to $8 \AA$ from absorbing copper atom. It was found that the dynamics of copper atoms is essentially anisotropic, in agreement with previous neutron diffraction studies [2], and the $\mathrm{Cu}-\mathrm{N}$ bonding is significantly covalent, as predicted by theoretical investigations [43]. Additionally, we have demonstrated that (i) motion of copper atom is strongly anharmonic and (ii) correlation effects play important role in the dynamics of neighboring atoms.

\section{Acknowledgments}

The work was supported by Latvian Science Council Grant No. 187/2012. The EXAFS experiments at HASYLAB/DESY were supported by the EC FP7 under grant agreement No. 226716 (Project I-20110160 EC).

\section{References}

[1] Caskey C M, Richards R M, Ginley D S and Zakutayev A 2014 Mater. Horizons 1 424-430

[2] Paniconi G, Stoeva Z, Doberstein H, Smith R I, Gallagher B L and Gregory D H 2007 Solid State Sci. 9 907-913

[3] Asano M, Umeda K and Tasaki A 1990 Jap. J. Appl. Phys. 29 1985-1986

[4] Maruyama T and Morishita T 1996 Appl. Phys. Lett. 69 890-891

[5] Ji Z, Zhang Y, Yuan Y and Wang C 2006 Mater. Lett. $603758-3760$

[6] Wang S, Qiu J, Wang X, Yuan N, Ding J and Huang W 2013 Appl. Surf. Sci. 268 387-390

[7] Zhao Y, Zhao J, Yang T, Zhang J, Yang J and Li X 2016 Ceram. Int. 42 4486-4490

[8] Borsa D M, Grachev S, Presura C and Boerma D O 2002 Appl. Phys. Lett. 80 1823-1825

[9] Fang Y, Persson J, Zha C, Willman J, Miller C W and Åkerman J 2012 J. Appl. Phys. 111073912

[10] Lu Q, Zhang X, Zhu W, Zhou Y, Zhou Q, Liu L and Wu X 2011 Phys. Status Solidi A 208 874-877

[11] Zhu W, Zhang X, Fu X, Zhou Y, Luo S and Wu X 2012 Phys. Status Solidi A 209 1996-2001

[12] Zhou Q, Lu Q, Zhou Y, Yang Y, Du X, Zhang X and Wu X 2013 Surf. Coat. Tech. 229 135-139

[13] Wu H and Chen W 2011 J. Am. Chem. Soc. 133 15236-15239

[14] Maya L 1993 J. Vacuum Sci. Tech. A 11 604-608

[15] Xu X, Yuan N, Qiu J and Ding J 2015 Mater. Res. Bull. 65 68-72

[16] Zakutayev A, Caskey C M, Fioretti A N, Ginley D S, Vidal J, Stevanovic V, Tea E and Lany S 2014 J. Phys. Chem. Lett. 5 1117-1125 
[17] Navio C, Capitan M J, Alvarez J, Yndurain F and Miranda R 2007 Phys. Rev. B 76085105

[18] Timoshenko J, Kuzmin A and Purans J 2013 J. Phys.: Conf. Ser. 430012012

[19] Timoshenko J, Kuzmin A and Purans J 2014 J. Phys.: Condens. Matter 26055401

[20] Rehr J J and Albers R C 2000 Rev. Mod. Phys. 72 621-654

[21] Kuzmin A and Chaboy J 2014 IUCrJ 1 571-589

[22] Provost K, Beret E C, Muller D, Marcos E S and Michalowicz A 2013 J. Phys.: Conf. Ser. 430 012015

[23] Okamoto Y 2004 Nucl. Instrum. Meth. Phys. Res. A 526 572-583

[24] Ferlat G, Soetens J C, Miguel A S and Bopp P A 2005 J. Phys.: Condens. Matter 17 S145

[25] Kuzmin A and Evarestov R A 2009 J. Phys.: Condens. Matter 21055401

[26] Yancey D F, Chill S T, Zhang L, Frenkel A I, Henkelman G and Crooks R M 2013 Chem. Sci. 4 2912-2921

[27] Metropolis N, Rosenbluth A W, Rosenbluth M N, Teller A H and Teller E 1953 J. Chem. Phys. 21 1087-1092

[28] McGreevy R L and Pusztai L 1988 Mol. Simul. 1 359-367

[29] Timoshenko J, Kuzmin A and Purans J 2012 Comp. Phys. Commun. 183 1237-1245

[30] Ankudinov A L, Ravel B, Rehr J J and Conradson S D 1998 Phys. Rev. B 58 7565-7576

[31] Filipponi A, Di Cicco A and Natoli C R 1995 Phys. Rev. B 5215122

[32] Kuzmin A, Anspoks A, Kalinko A and Timoshenko J 2015 Z. Phys. Chem. doi: 10.1515/zpch2015-0664

[33] Gale J D 1996 Phil. Mag. B 73 3-19

[34] Berne B J and Thirumalai D 1986 Ann. Rev. Phys. Chem. 37 401-424

[35] Rickers K, Drube W, Schulte-Schrepping H, Welter E, Brüggmann U, Herrmann M, Heuer J and Schulz-Ritter H 2007 AIP Conf. Proc. 882 905-907

[36] Aksenov V L, Koval'chuk M V, Kuz'min A Y, Purans Y and Tyutyunnikov S I 2006 Cryst. Rep. 51 908-935

[37] Kuzmin A 1995 Physica B 208-209 175-176

[38] Kuzmin A, Purans J, Benfatto M and Natoli C R 1993 Phys. Rev. B 47 2480-2486

[39] Funke H, Scheinost A C and Chukalina M 2005 Phys. Rev. B 71094110

[40] Timoshenko J and Kuzmin A 2009 Comp. Phys. Commun. 180 920-925

[41] Kalinko A, Evarestov R A, Kuzmin A and Purans J 2009 J. Phys.: Conf. Ser. 190012080

[42] Gale J D and Rohl A L 2003 Mol. Simul. 29 291-341

[43] Hahn U and Weber W 1996 Phys. Rev. B 53 12684-12693

[44] Abrahamson A A 1969 Phys. Rev. 17876

[45] Hoover W G 1985 Phys. Rev. A 31 1695-1697

[46] Hockney R W 1970 Methods Comput. Phys. 9 136-211

[47] Fornasini P 2001 J. Phys.: Condensed Matter 13 7859-7872

[48] Jonane I, Lazdins K, Timoshenko J, Kuzmin A, Purans J, Vladimirov P, Gräning T and Hoffmann J 2016 J. Synchrotron Rad. 23 510-518

[49] Zachwieja U and Jacobs H 1990 J. Less Common Metals 161 175-184

[50] Timoshenko J, Kuzmin A and Purans J 2015 J. Phys.: Conf. Ser. (in press)

[51] Vajenine G V, Hoch C, Dinnebier R E, Senyshyn A and Niewa R 2010 Z. Anorg. Allg. Chem. 636 94-99 